\title{
Regulation Roles of MICA and NKG2D in Human Renal Cancer Cells
}

\author{
Hong-Ying Jia ${ }^{1}$, Jun-Li Liu ${ }^{1}$, Ming-Zhen Yuan ${ }^{2}$, Cheng-Jun Zhou ${ }^{3}$, Wen-Dong \\ Sun $^{2}$, Jing-Jie Zhao ${ }^{1}$, Jue Wang ${ }^{4}$, Ling Liu ${ }^{1}$, Yun Luan ${ }^{4 *}$
}

\begin{abstract}
Objective: Our aim was to investigation the roles of MHC class I chain-related gene A(MICA) and natural killer cell group 2D(NKG2D) in human renal cancer cells. Materials and Methods: The expression of membrane MICA (mMICA) on renal cells and NKG2D on NK cells were detected by flow cytometry (FCM); the content of sMICA were detected by enzyme linked immunosorbent assay (ELISA) and the distribution of mMICA on renal tumor tissues by immunohistochemistry; the interaction between MICA and NKG2D was observed by antibody closed method. Results: Our results showed that the expression of mMICA in renal cancer tissues was significantly higher than in controls, where the soluble MICA was not expressed. Cytotoxic activity of NK cells was significantly reduced after exposure to NKG2D and MICA antibodies $(P<0.05)$, and serum containing SMICA can obviously lower the function of NKG2D $(P<0.05)$. Conclusions: The interaction of mMICA and NKG2D play important roles in mediation of cytotoxicity of NK cells in RCC. On the other hand, sMICA may mediate tumor immune escape through down- regulated NKG2D expression.
\end{abstract}

Keywords: Renal cell carcinoma - MICA - NKG2D - NK cells - cytotoxicity

Asian Pac J Cancer Prev, 16 (9), 3901-3905

\section{Introduction}

Natural killer cells (NK cells) are a type of cytotoxic lymphocyte capable of mediating early innate immune responses to viral infections and recognition of transformed malignant cells (Diefenbach et al., 2002; Bae et al., 2014). The cytolytic activity of NK cells against tumor cell lines was recognized very early and it is now becoming evident that several characteristics of tumor cells may induce NK cell-mediated antitumor reactivity (Doubrovina et al., 2003 ; Coursey et al., 2014; Wang et al., 2014). NKG2D is an activating receptor of natural killer cells newly discovered in recent years, which is thought to play an important role in tumor immune surveillance (Lanier, 2001; Matusali et al., 2013; Cho et al., 2014). Approximately $90 \%$ of RCC lesions express MHC class I antigens, in recent years, some highly diversified MHCI-related molecules MICA/B are major ligands of NKG2D and are often expressed in tumor cells (La Scaleia et al., 2012; Meyer et al., 2014). NK cells can recognize tumor cells that have downregulated MHC class I Ag expression (Bae et al., 2012). Through NKG2D, NK cells may identify the target cells structures, such as MHC class I chain-related molecule A (MICA) and MICB, that normally are not expressed or are present in very low density but become up-regulated in transformed cells (Doubrovina et al., 2003; Chen et al., 2014). The relationship of expression of NKG2D and MICA in the tumor microenvironment by inflammatory cells in RCC specimens have not clearly been investigated so far.

In our early study, we have confirmed that high expression of MICA in human kidney cancer and MICA might serve as potential tumor associated antigen in RCC (Jia et al., 2014). Further analysis the significance and clinical relevance of NKG2D and MICA as well as their underlying molecular mechanisms in RCC lesions will contribute to the optimization of T-cell-based immunotherapeutic strategies for the treatment of RCC patients.

\section{Materials and Methods \\ Cell lines and tissue specimen}

Cell lines (A498, 786-O, Ketr-3 and HEK293) were obtained from Chinese Academy of Medical Sciences. Which were cultured in Dulbecco's modified Eagle's medium with $10 \%$ fetal bovine serum (Hyclone, USA). Human RCC specimens and normal tissues were collected from patients who underwent surgery according to an approved human protocol at the Second Hospital and Qilu Hospital of Shandong University (China).

Isolation, purification and identification of NK cell 
The NK cells were isolated and purification through Ficoll separation method as our previously reports (Jia et al., 2014). Vario-MACS system magnetic bead separation kit (Miltenyi Company, Germany) were used to purification of NK Cells and the percentage of CD3CD56+ cells were determined by flow cytometry.

\section{Expression of mMICA in RCC cell lines and tissue specimen}

mMICA expression in kidney cancer were detection by FCM , immunohistochemical and reverse transcriptionpolymerase chain reaction (RT-PCR) as previously report. Briefly, $10 \mu \mathrm{l}$ MICA-PE labeling antibody was plused in to $1 \times 10^{6}$ cells/well (A498, 786-O, Ketr-3 and HEK293 cell lines) using flow cytometry instrument (FACS LSRFortessa, BD) for testing. Total RNA was extracted from kidney tissue using a Qiagen (Basel, Switzerland) RNeasy kit. Complementary DNA (cDNA) first strand was produced using a SuperScript First-Strand Synthesis System using oligo (dt) antisense primers (Invitrogen, Lucerne, Switzerland). Amplified fragments were analyzed in $1.5 \%$ agarose gel electrophoresis in the presence of ethidium bromide (Sigma). $\beta$-actin (500 bp) was used as an internal control for the amount of RNA input.

Human kidney tissues in healthy and cancer patients were fixed in $10 \%$ formaldehyde, and then were embedded in paraffin, sections were cut into $5 \mu \mathrm{m}$ slices and were stained with mMICA (1:100, Santa Cruz Biotechnology) antibody under the manufacturer's. The sections were observed and photographed with a Nikon Eclipse 90i microscope.

NKG2D- and mMICA-mediated NK cell cytotoxicity activity was detected by MTT method

Each RCC cell line during the logarithmic phase of growth were the target cells (T), purified NK cells was effector cells (E). The cells were maintained in continuous suspension culture at 96 cell culture plates at $37^{\circ} \mathrm{C}$ in an atmosphere of $5 \% \mathrm{CO}_{2}$ incubator. Blank group: only medium; Target group: various RCC cells; Effector group: NK cells; Experimental group: NK cells + RCC cells. Each group for four holes, A final concentration of the target cells in the complete culture medium was $5 \times 10^{5}$ and the ratio of E:T was 10:1. The MTT (Sigma, USA) solution of $5 \mathrm{mg} / \mathrm{mL}$ was added to the cultures containing $(10 \mu \mathrm{L}$ MTT solutions per $100 \mu \mathrm{L}$ medium) and the cultures were incubated for $20 \mathrm{~h}$ at $37^{\circ} \mathrm{C}$ in $5 \% \mathrm{CO}_{2}$. Optical density (OD) of each culture was read by Universal Microplate Reader at wave length of 570nm.

10 healthy adult peripheral blood, purification of the NK Cells by the above mentioned method, NKG2D was blocked by NKG2D antibody $(5 \mu \mathrm{g} / \mathrm{m} 1)$ and MICA was blocked by MICA antibody $(10 \mu \mathrm{g} / \mathrm{m} 1)$. The cells were placed in the ice water for 30 minutes and PBS washing twice, after NKG2D-APC and MICA-PE staining, the closed effect was detected by FCM, and NK cell cytotoxicity was determined by MTT method.

Effect of SMICA on expression of NKG2D and NK cell cytotoxicity
The content of sMICA in serum were determined by enzyme linked immunosorbent assay (ELISA) as previously described with some modifications (Jia et al., 2014), briefly, human MICA antibody was added in 1/100 dilution to the plate precoated with PBS and incubated forlh at room temperature. A HRP-conjugated goat antirabbit IgG (Baso Biotechnology, ShenZhen, China) was used as the secondary antibody. The reactions were read at adsorption wavelengths of $450 \mathrm{~nm}$ and $570 \mathrm{~nm}$ using a microplate reader (SAFIRE, Tecan, Austria). To observe sMICA effect on the activity of NK cells to kill tumor, we co-cultureed NK cells with sMICA. The groups as the following progress: healthy serum+healthy NK cell; sMICA (-) serum+ healthy NK cell; sMICA (+) serum thealthy NK cell; sMICA $(+)$ serum + SMICA $(+)$ NK cell. We collected NK cells after co-culture the cells at 12,15, 22,40 , and $48 \mathrm{~h}$, the expression of NKG2D was detected by FCM and the effect of sMICA on NK cell cytotoxicity were detected by MTT.

\section{Statistical analysis}

All data are expressed as mean \pm SEM. Comparisons of parameters between 2 groups were made with unpaired Student $t$ test. Comparisons of parameters among 3 groups were made with one-way analysis of variance (ANOVA), followed by the Scheffe multiple-comparison test. Statistical analysis was carried out by using the SPSS 13.0 software. $P<0.05$ was regarded as significant statistical difference.

\section{Results}

mMICA expression in tumor cell lines and tissue specimen

Flow cytometry detection results showed that there are almost no expression of mMICA in human embryonic kidney 293 (HEK293) cell line and low expression in RCC cell line A498 (7.47\%), but the expression level was significantly increased in RCC cell lines 786-O (67.16\%) and Ketr-3 (25.7\%) individually (Figure 1).

\section{Expression of mMICA in human kidney cancer tissue}

Immunohistochemical detection of kidney tissue specimens of mMICA expression also showed that significangly high in kidney cancer group than in healthy group $(11.3 \% \pm 1.6 \%$ vs $69.8 \% \pm 2.9 \%$; $\mathrm{P}<0.05,48$ case in each group, Figure 2).

\section{Soluble MICA (sMICA) detection}

ELISA analysis showed that the content of sMICA in serum of healthy was negative, but there was $70.8 \%$ (34/48) positive in kidney cancer patients, the content was (196.35 50.27) $\mathrm{pg} / \mathrm{ml}$.

\section{NK cell cytotoxicity activity}

FCM results showed that after NKG2D antibody blocked, the expression was significangly lower than unblochade $(1.67 \%$ vs $90.62 \%, P<0.01)$; the MICA expression in $786-\mathrm{O}(1.94 \%)$ and Ketr-3 $(0.82 \%)$ were also significantly lower after blocked than befoer $(67.16 \%$ and $25.37 \%, P<0.05)$. There was no significantly change in A498 cell line which was low expression of MICA. These 


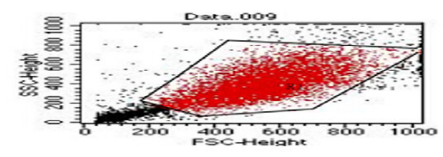

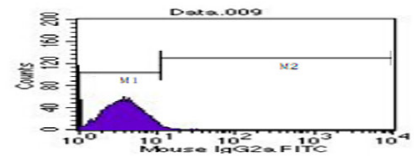

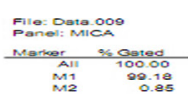
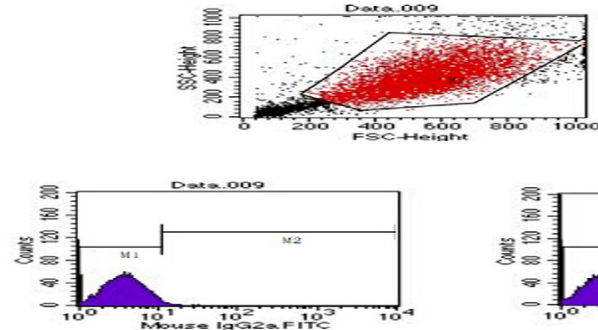

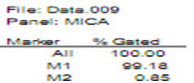
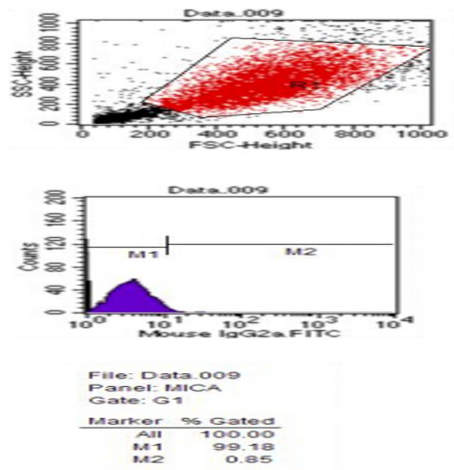
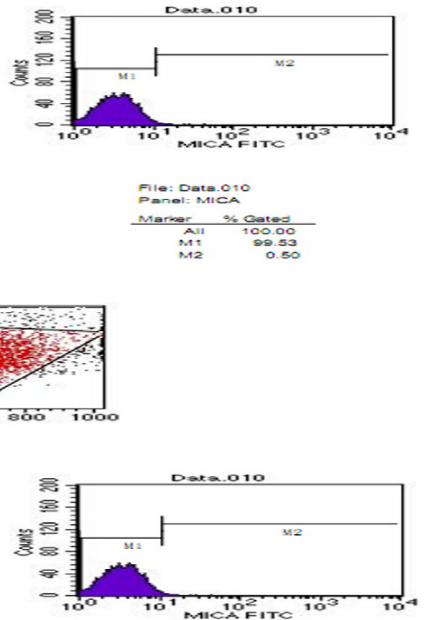

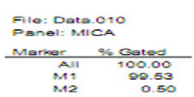
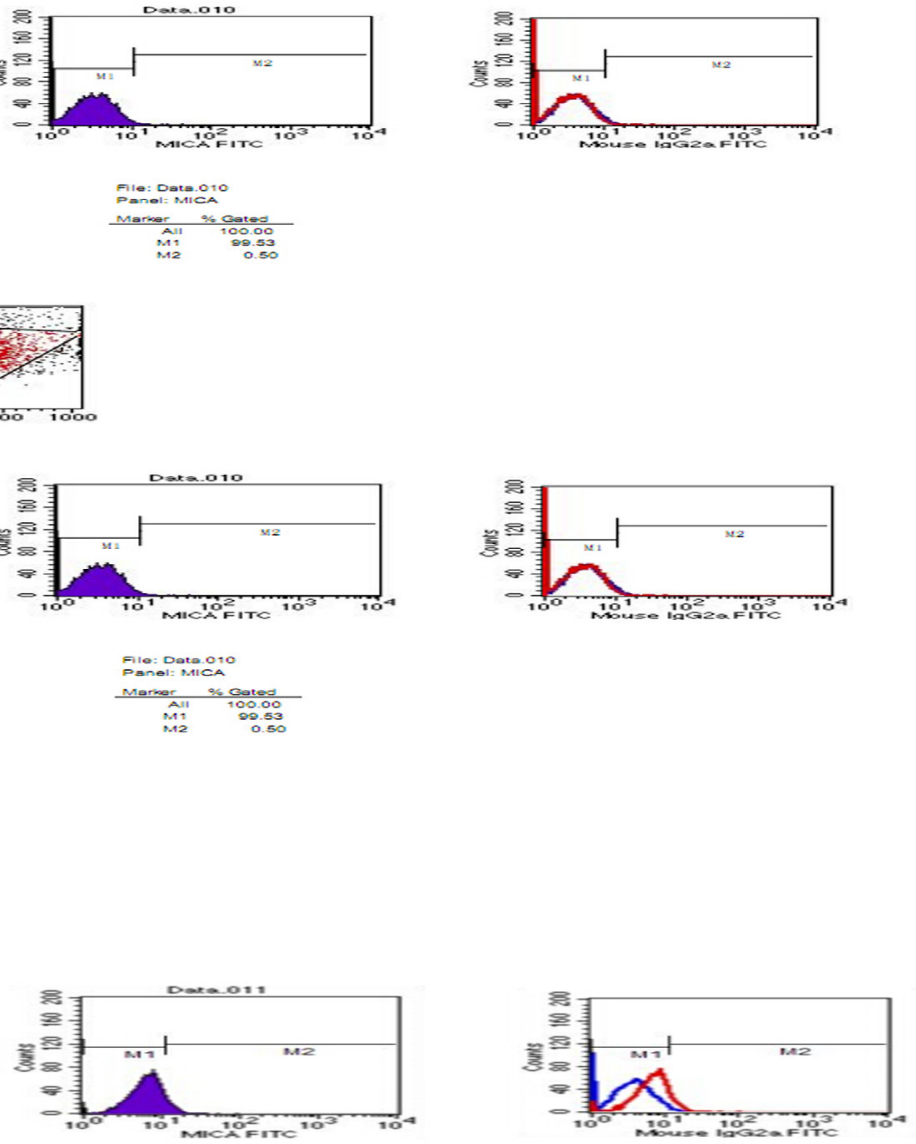

Figure 1. mMICA Expression in Tumor Cell Lines and Tissue Specimen. A) H293, B) 786-O, C) ketr-3, D) A498

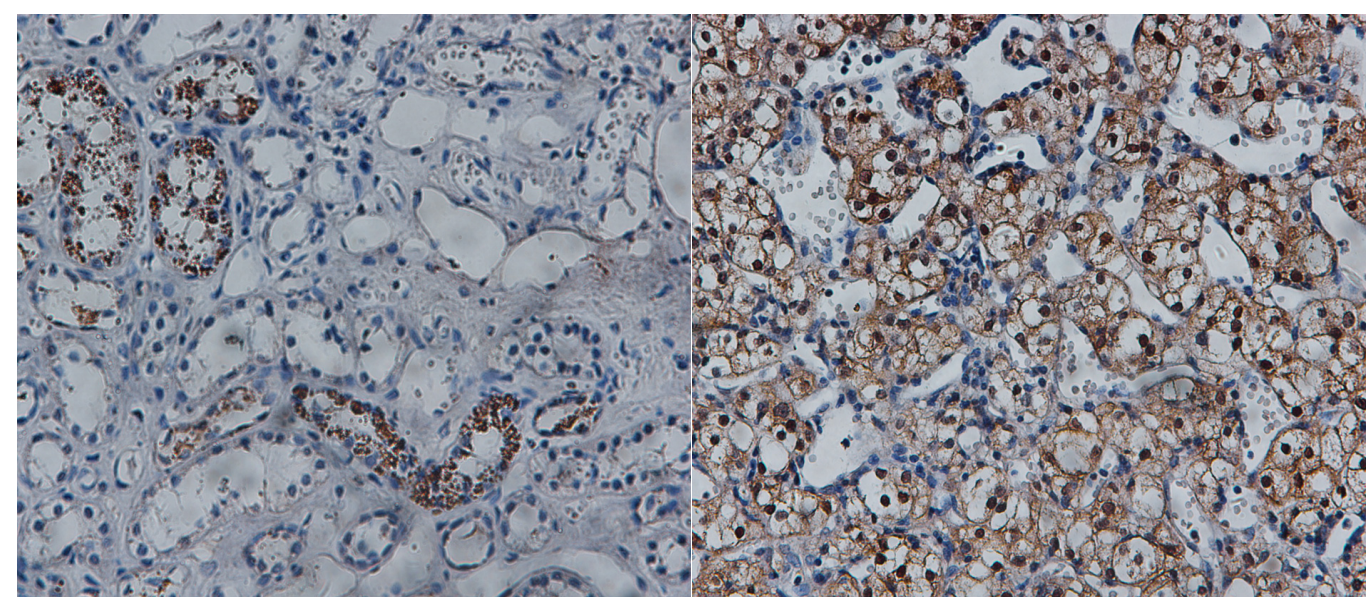

Figure 2. Expression of mMICA in Human Kidney Cancer Tissue. A) healthy kidney, B) Cancer

indicated that the NKG2D site in NK cell and MICA in RCC cell lines (786-O and Ketr-3) were visible blocked.

At the same time, we used MTT method to detect the NK cell cytotoxicity activity, the results showed that when NK cells were b1ocked by NKG2D antibody, the activity were significantly reduced than unblocked, the activity of NK cells to 786-O and Ketr-3 which was blocked by MICA antibody but not blocked by NKG2D were significantly reduced than not blocked by MICA antibody, but the NK cells activity in A498 (low expression of MICA) almost no change $(P>0.05)$. The cytotoxicity activity was reduced more significantly when NK cells were b1ocked by NKG2D antibody and RCC cells were blocked by MICA antibody (Figure 3). 


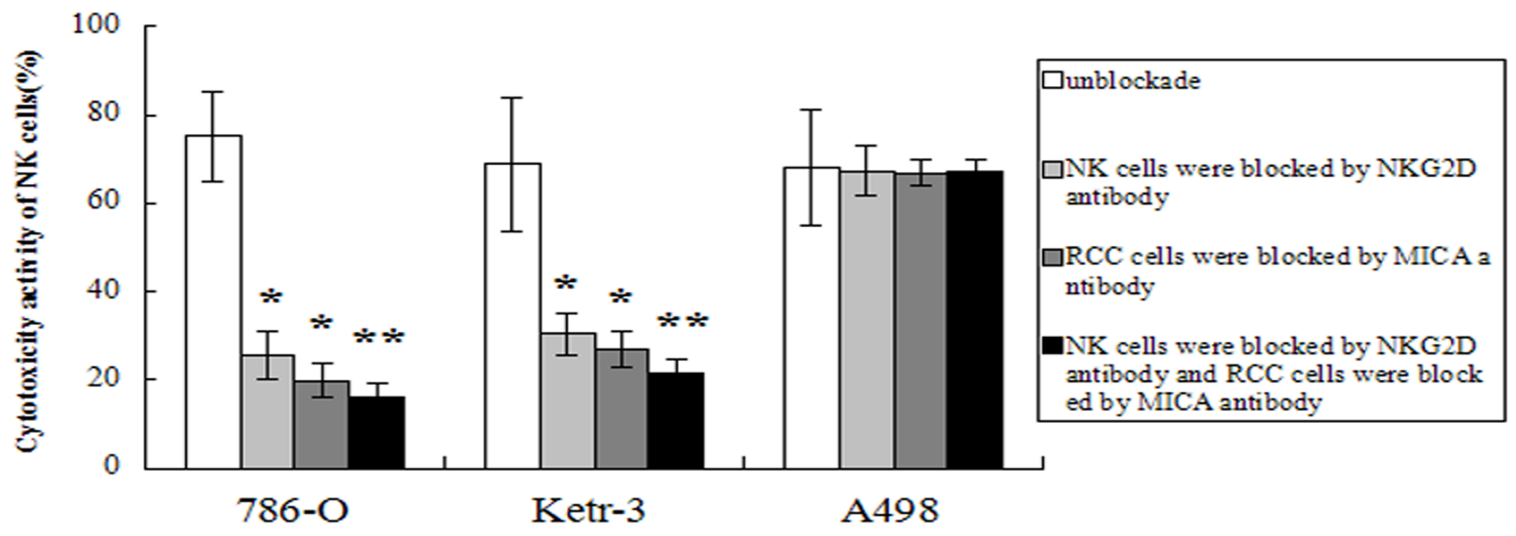

Figure 3. NK Cell Cytotoxicity Activity. NK cells were b1ocked by NKG2D antibody, the activity were significantly reduced than unblockade, the activity of NK cells to 786-O and Ketr-3 which was blocked by MICA antibody but not blocked by NKG2D were significantly reduced than not blocked by MICA antibody, but the NK cells activity in A498 (low expression of MICA) almost no change. The cytotoxicity activity was reduced more significantly when NK cells were b1ocked by NKG2D antibody and RCC cells were blocked by MICA antibody. Data expressed as mean of mean \pm standard error $\pm \mathrm{SE}$. \#P $<0.05, * * \mathrm{P}<0.01$ compared with unblocked group

Table 1. The Effect of sMICA on Cytotoxicity Activity of NK Cells and Expression of NKG2D (24h)

\begin{tabular}{lccccc}
\hline Groups & \multicolumn{2}{c}{$\begin{array}{c}\text { Expression of } \\
\text { NKG2D }(\%)\end{array}$} & & \multicolumn{2}{c}{$\begin{array}{c}\text { Cytotoxicity } \\
\text { activity }(\%)\end{array}$} \\
\cline { 2 - 3 } \cline { 5 - 6 } & $\begin{array}{c}\text { Pre- } \\
\text { cultivation }\end{array}$ & $\begin{array}{c}\text { Post- } \\
\text { cultivation }\end{array}$ & & $\begin{array}{c}\text { Pre- } \\
\text { cultivation }\end{array}$ & $\begin{array}{c}\text { Post- } \\
\text { cultivation }\end{array}$ \\
\hline I & $80.7 \pm 11.5$ & $81.6 \pm 12.3$ & & $94.6 \pm 12.6$ & $93.8 \pm 10.8$ \\
II & $79.8 \pm 10.6$ & $69.4 \pm 11.7$ & & $95.2 \pm 11.4$ & $85.6 \pm 9.8$ \\
III & $80.5 \pm 12.4$ & $50.2 \pm 10.6^{*}$ & & $94.3 \pm 10.7$ & $65.2 \pm 11.2 *$ \\
IV & $56.8 \pm 13.2$ & $55.6 \pm 11.5$ & & $78.4 \pm 13.5$ & $75.3 \pm 12.7$ \\
\hline
\end{tabular}

I:Normal serum+normal NK cells; II: sMlCA(-)palients srum+normal NK cells; III: sMICA(+)patients serum+normal NK cells; IV:sMICA(+) patiems serum+sMICA(+)

Effect of sMICA on activity of NK cells and expression of $N K G 2 D$

Our results showed that the expression of NKG2D on the surface of NK cells in $15 \mathrm{~h}$ began to decline, the lowest in 24-40 h. At the same time (24h) to testing activity of NK cells to kill RCC cells significantly decreased (Table 1).

\section{Discussion}

In the present study, we evaluated the regulation role of MICA and NKG2D in human renal cancer cells, our research supports a central role for interaction of mMICA and NKG2D play an important role in mediation the cytotoxic of NK cells in RCC.

Renal cell carcinoma is the most common type of kidney cancer and follows an unpredictable disease course (Noon et al., 2010), represents approximately 2\% -3\% in all malignant tumor, a median survival of approximately 1 yearb (Cohen et al., 2005; Sconocchia et al., 2009). Annual incidence increased at about 2\%, nearly 100,000 cases of patients died of kidney cancer each year. The particular resistance to many therapeutic approaches, such as conventional chemotherapy or radiotherapy, is the main cause of dismal prognosis (Weissinger et al., 2013; Yang et al., 2013). Thus, further exploration and alternative treatment strategies are highly need. Natural killer cells are a type of cytotoxic lymphocyte capable of mediating early innate immune responses to viral infections and recognition of transformed malignant cells. The cytolytic activity of NK cells against tumor cell lines was recognized very early and it is now becoming evident that several characteristics of tumor cells may induce NK cell-mediated antitumor reactivity. NKG2D is an activating receptor of natural killer cells newly discovered in recent years, which is thought to play an important role in tumor immune surveillance (Matusali et al., 2013; Cho et al., 2014). Approximately $90 \%$ of RCC lesions express MHC class I antigens, in recent years, some highly diversified MHCI-related molecules MICA/B are major ligands of NKG2D and are often expressed in tumor cells. NK cells can recognize tumor cells that have down-regulated MHC class I Ag expression (Wang et al., 2014; Coursey et al., 2014). Through NKG2D, NK cells may identify the target cells structures, such as MHC class I chain-related molecule A (MICA) and MICB, that normally are not expressed or are present in very low density but become up-regulated in transformed cells. The relationship of expression of NKG2D and MICA/B in the tumor microenvironment by inflammatory cells in RCC specimens have not clearly been investigated so far. Further analysis the significance and clinical relevance of NKG2D and MICA/B as well as their underlying molecular mechanisms in RCC lesions will contribute to the optimization of T-cell-based immunotherapeutic strategies for the treatment of RCC patients.

In our previously study (Jia HY et al., 2014), we also indicated that the percentage of mMICA expression was significantly increased in human kidney cancer tissues and RCC cell lines (786-O and Ketr-3) than that in healthy adults and human embryonic kidney 293 (HEK293) cell line individuality; sMICA content in healthy adults were negative, but in renal cancer patients was significantly higher.

In this study FCM results showed that after NKG2D antibody blocked, the expression was significantly lower than unblochade; the MICA expression in 786-O and Ketr-3 were also significantly lower after blocked than 
before. There was no significantly change in A498 cell line which was low expression of MICA. These indicated that the NKG2D site in NK cell and MICA in RCC cell lines were visible blocked. At the same time when NK cells were b1ocked by NKG2D antibody, the activity were significantly reduced than unblockade, the activity of NK cells to 786-O and Ketr-3 which was blocked by MICA antibody but not blocked by NKG2D were significantly reduced than not blocked by MICA antibody, but the NK cells activity in A498 (low expression of MICA) almost no change. The cytotoxicity activity was reduced more significantly when NK cells were b1ocked by NKG2D antibody and RCC cells. All these results showed that the interaction of mMICA and NKG2D play an important role in mediation the cytotoxic of NK cells in RCC. On the other hand, sMICA mediated tumor immune escape through down- regulated NKG2D expression.

\section{Acknowledgements}

This study was supported by grants from the Jinan Science and Technology Development Projects (201218005) and Natural Science Foundation of Shandong Province ( ZR2013HM103 and ZR2014HQ045).

\section{References}

Bae DS, Lee JK (2014). Development of NK cell expansion methods using feeder cells from human myelogenous leukemia cell line. Blood Res, 49, 154-61.

Bae DS, Hwang YK, Lee JK (2012). Importance of NKG2DNKG2D ligands interaction for cytolytic activity of natural killer cell. Cell Immunol, 276, 122-7.

Coursey TG, Bohat R, Barbosa FL, Pflugfelder SC, de Paiva CS (2014). Desiccating stress-induced chemokine expression in the epithelium is dependent on upregulation of NKG2D/ RAE-1 and release of IFN- $\gamma$ in experimental dry eye. $J$ Immunol, 15, 5264-72.

Cho H, Chung JY, Kim S, et al (2014). MICA/B and ULBP1 NKG2D ligands are independent predictors of good prognosis in cervical cancer. BMC Cancer, 14, 957-61.

Chen D, Gyllensten U (2014). MICA polymorphism: biology and importance in cancer. Carcinogenesis, 35, 2633-42.

Cohen HT, McGovern FJ (2005). Renal-cell carcinoma. N Engl J Med, 353, 2477-90.

Diefenbach A, Raulet DH (2002). The innate immune response to tumors and its role in the induction of T-cell immunity. Immunol Rev, 188, 9-21.

Doubrovina ES, Doubrovin MM, Vider E, et al (2003). Evasion from NK cell immunity by MHC classes I chain-related molecules expressing colon adenocarcinoma. J Immunol, 171, 6891-9.

Jia HY, Liu JL, Zhou CJ, et al (2014). High expression of MICA in human kidney cancer tissue and renal cell carcinoma lines. Asian Pac J Cancer Prev, 15, 1715-17.

La Scaleia R, Stoppacciaro A, Oliva S, et al (2012). NKG2D/ Ligand dysregulation and functional alteration of innate immunity cell populations in pediatric IBD. Inflamm Bowel Dis, 18, 1910-22.

Lanier LL (2001).Arenaissance for the tumor immunosurveillance hypothesis. Nat Med, 7, 1178-80.

Matusali G, Tchidjou HK, Pontrelli G, et al (2013). Soluble ligands for the NKG2D receptor are released during HIV-1 infection and impair NKG2D expression and cytotoxicity
DOI:http://dx.doi.org/10.7314/APJCP.2015.16.9.3901 Roles of MICA-NKG2D in Human Renal Cell Cancer Cells of NK cells. FASEB J, 27, 2440-50.

Meyer A, Carapito R, Ott L, et al (2014). High diversity of MIC genes in non-human primates. Immunogenetics, 66, 581-7.

Noon AP1, Vlatković N, Polański R, et al (2010). p53 and MDM2 in renal cell carcinoma:biomarkers for disease progression and future therapeutic targets? Cancer, 116, 780-90.

Raulet DH (2003). Roles of the NKG2D immuno-receptor and its ligands. Nat Rev Immunol, 3, 781-90.

Sconocchia G, Spagnoli GC, Del Principe D, et al (2009). Defective infiltration of natural killer cells in MICA/Bpositive renal cell carcinoma involves beta (2)-integrin mediated interaction. Neoplasia, 11, 662-71.

Wang W, Guo H, Geng J, et al (2014). Tumor-released Galectin-3, a soluble inhibitory ligand of human NKp30, plays an important role in tumor escape from NK cell attack. J Biol Chem, 289, 33311-9.

Weissinger D, Tagscherer KE, Macher-Goppinger S, et al (2013). The soluble Decoy receptor 3 is regulated by a PI3K-dependent mechanism and promotes migration and invasion in renal cell carcinoma. Mol Cancer, 12, 120-35.

Yang F, Shao Y, Yang F, et al (2013). Valproic acid upregulates NKG2D ligand expression and enhances susceptibility of human renal carcinoma cells to NK cell mediated cytotoxicity. Arch Med Sci, 9, 323-31. 